\title{
Biblioteca pública y acceso a la información: retos para la sociedad de la información en Brasil y España
}

\author{
Maria Cleide Rodrigues Bernardino \\ Universidade Federal do Cariri - UFCA, Brasil
}

Aurora Cuevas-Cerveró

Universidad Complutense de Madrid - UCM, España

Emir José Suaiden

Universidade de Brasília - UnB, Brasil

\section{ANALYSIS}

\begin{abstract}
Resumen
Objetivo. Presenta una discusión teórica sobre la concepción de la biblioteca como una institución democrática que se relaciona directamente con el derecho a la información, la cual garantiza el acceso al conocimiento, la cultura, la educación y el ocio de acuerdo con sus funciones y al Manifiesto de la UNESCO. Método. Este es un estudio exploratorio con análisis cualitativo, cuantitativo y metodo dialéctico. Fue realizado en bibliotecas públicas de Brasil y España, observando los aspectos de la interactividad y conectividad, como supuestos necesarios para la sociedad de la información. Resultados. El fundamento teórico se basa en estudios de Suaiden (2012); Felicia Soto (2006); Jaramillo, et al (2008 los cuales introducen cuestiones sobre la misión de la biblioteca pública y los retos para la sociedad de la información; además Cuevas-Cerveró (2009) y Cuevas-Cerveró y Simeão (2011), quien señalan la participación de la biblioteca pública como lugar de formación para el aprendizaje informacional. Conclusiones. La sociedad de la información requiere el libre acceso a la información a todos aquellos que lo necesitan en cualquier soporte o formato, incluyendo el electrónico. Sin embargo, aun tenemos desigualdades sociales responsables de la brecha digital.
\end{abstract}

Palabras clave

Biblioteca pública ; Acceso a la información ; Sociedad de la información ; Brasil ; España

\section{Public library and information access: challenges for the information society in Brazil} and Spain

\begin{abstract}
Objective. It presents a theoretical discussion of the concept of the library as a democratic institution that is directly related to the right to information, which guarantees access to knowledge, culture, education and leisure according to their functions and the Manifesto UNESCO. Method. This is an exploratory study with qualitative, quantitative analysis and dialectical method. It was made in public libraries in Brazil and Spain, noting aspects of interactivity and connectivity, as assumptions necessary for the information society. Results. The theoretical framework is based on studies of Suaiden (2012); Felicia Soto (2006); Jaramillo, et al (2008 which introduced questions about the mission of the public library and the challenges for the information society, plus Cuevas-Cerveró (2009) and Cuevas-Cerveró and Simeon (2011), who point to the involvement of public library as a place of training for informational learning. Conclusões. The information society requires free access to information to those who need it in any medium or format, including electronic. However, we still have social inequalities responsible for the digital divide.
\end{abstract}




\section{Introducción}

Este trabajo presenta una discusión teórica acerca de la concepción de la biblioteca pública como una institución democrática y que se relaciona directamente con el derecho a la información, que por su parte debe garantizar el acceso al conocimiento, la cultura, la educación y el ocio de acuerdo con sus funciones y a los dictados del Manifiesto de la UNESCO.

En la sociedad contemporánea - llamada sociedad de la información - es grande el volumen, la velocidad de la producción de la información y el flujo de la información y del conocimiento. El valor social de la información y su subjetividad ayudó a determinar el derecho a la socialización de conocimientos y por lo tanto, su uso arraiga al sujeto en la sociedad y garantiza el ejercicio pleno de su ciudadanía. Couto (1988, p. 66) establece que la información se "identifica como una necesidad social" muy importante para la actividad humana. Y a la biblioteca pública le corresponde garantizar a los ciudadanos el derecho a la información necesaria para su existencia.

Conforme el Manifiesto de la UNESCO de 1994 la biblioteca pública debe actuar de acuerdo con "la libertad, la prosperidad y el desarrollo de la sociedad y de la persona" pues estos son "valores humanos fundamentales que sólo podrán alcanzarse si ciudadanos bien informados pueden ejercer sus derechos democráticos y desempeñar un papel activo dentro de la sociedad". Sin embargo de acuerdo con el Manifiesto la "participación constructiva y la consolidación de la democracia dependen de una buena educación y de un acceso libre e ilimitado al conocimiento, el pensamiento, la cultura y la información". Hemos dicho que la sociedad de la información requiere el libre acceso a la información a todos aquellos que lo necesitan en cualquier soporte o formato y en este sentido la biblioteca pública es la institución que reúne las condiciones ideales para facilitar el acceso a la información para las personas que la necesitan en la sociedad.

Sin duda los servicios que ofrece la biblioteca pública deben incluir todos los tipos de medios y tecnologías modernas y también materiales tradicionales, a fin de garantizar el acceso a la información en cualquier lugar, por cualquier persona y en cualquier momento. En este sentido los retos para la biblioteca pública en la sociedad de la información son muy grandes e incluyen los aspectos relacionados con la brecha digital y la aprendizaje informacional.

\section{La misión de la biblioteca pública y los retos para la sociedad de la información}

Miranda (1978, p. 2) expone que "la biblioteca pública es un fenómeno histórico bajo una influencia recíproca y permanente (interacción) con el medio ambiente" y al involucrar a las personas en su organización y control de la información, tales como bibliotecarios y ayudantes se convierte en una célula viva. Esta interacción constante requiere que la misión de la biblioteca pública vaya más allá de las leyes que rigen y programa? En este sentido, la misión de la biblioteca pública es principalmente atender a la comunidad a cual pertenece proporcionando información en cualquier formato o medio. Esto significa que en tiempos de innovación tecnológica y medios digitales modernos las bibliotecas públicas enfrentan retos cada vez más grandes y más complejos.

Por lo tanto, se amplía el alcance de la misión de la biblioteca pública en la sociedad de la información, ya que según Jaramillo, et al (2008, p. 9) los "profundos cambios económicos, políticos, sociales, culturales, educativos, tecnológicos e informativos que se han vivido, especialmente en las últimas dos décadas" son responsables a la hora de cambiar también la realidad y las perspectivas de las bibliotecas públicas en la sociedad.

Al respecto Martínez (2002, p. 144) expone,

[...] la sociedad de la información, a la vez que ofrece nuevas posibilidades, pone en tensión muchas parejas de elementos que son fundamentales para la biblioteca pública: tradición y modernidad, abundancia de datos disponibles y datos significativos, diversidad y normalización, información y conocimiento, y algunos más que podríamos enumerar.

Los cambios en la sociedad y en el flujo de información mediado por las tecnologías afectan a las bibliotecas públicas y sus servicios, lo cual, en un contexto informacional de profundas transformaciones, revela una nueva función para la biblioteca pública como productora y difusora de información digital. Por otra parte, esta nueva misión tiene retos aún mayores en cuanto a la gestión económica y política en las bibliotecas públicas. Acerca de esta cuestión Azevedo y Gouveia (2003, p. 121) dicen que,

La respuesta a este nuevo reto debe adoptar la forma de estrategias que, en la práctica de nuevas habilidades y servicios, ayuden a mantener y ampliar el alcance de la misión de la biblioteca, como mediadora y facilitadora de la difusión de los conocimientos a la comunidad. 
Esto significa que el mayor reto para la biblioteca pública en la sociedad de la información es proponer estrategias que permitan a sus clientes utilizar la información digital, incluso con todas las limitaciones de la brecha digital. Seoane-Garcia (2012) plantea que la internet está revolucionado el mundo y por consiguiente el medio y la forma en que las personas acceden, consumen y comparten informaciones. Esto nos sitúa ante un nuevo concepto de biblioteca pública que puede entenderse como un ambiente de aprendizaje informacional, conforme declara Seoane-Garcia (2012, p. 1):

En la actualidad ya nadie duda del papel que desempeñan las nuevas tecnologías en las bibliotecas, convirtiendo a éstas en verdaderos instrumentos de comunicación y alfabetización, lugares propicios para romper el latente aumento de la brecha digital entre generaciones y otorgar a los ciudadanos la posibilidad de tener acceso equitativo a la información y al conocimiento.

Por un lado las nuevas tecnologías amplían el campo de actuación de las bibliotecas públicas pero también las limitan, pues en tiempos de acceso y derecho a la información las instituciones que no estén conectadas no podrán actuar con eficacia en la sociedad de la información y el conocimiento. La amplitud de las funciones de las bibliotecas públicas generadas por la conectividad demanda actividades de aprendizaje informacional, dado que para combatir la brecha digital es necesario un buen desempeño de las instituciones que trabajan con información.

\section{Aprendizaje informacional en la biblioteca pública}

Con las innovaciones tecnológicas el acceso a la información se ha expandido, lo que al mismo tiempo que se constituye en una ventaja para la biblioteca pública, también se convierte en una desventaja por llevar al requisito de nuevos y mayores retos y responsabilidades. Otro factor muy importante es la competencia informacional de los profesionales que trabajan en estas bibliotecas. Seoane-Garcia (2012, p. 2) hablando acerca de la crisis y recesión en España que ha afectado a todos los servicios incluso los servicios informacionales, o sea, la biblioteca pública, argumenta que se, "hace necesario que la Biblioteca Pública cumpla un papel catalizador, que adecue su actuación al contexto actual y a las necesidades reales de sus usuarios y que la evidente reducción presupuestaria se compense con un nuevo enfoque de servicios".

Tomando en préstamo las palabras de Seoane-Garcia (2012) aunque traten de herramientas de la Web 2.0, entendemos que la biblioteca debe preocuparse con los aspectos de la brecha digital y ofrecer servicios que puedan minimizar estos problemas. Esta fue una preocupación de instituciones y personas que se refleja en diferentes debates y manifiestos, como la Declaración de Copenhague, en 1999, que ha discutido el papel clave y las funciones de las bibliotecas públicas en las sociedades emergentes de la información, donde se destacó su responsabilidad con la alfabetización informacional y el aprendizaje permanente (DÍAZ-SÁNCHEZ, 2008).

El sintagma alfabetización informacional ha tenido distintos significados a través del tempo y tiene relación con competencias y habilidades, en este sentido Díaz-Sánchez, 2008, p. 111) observa que "se relaciona con el conjunto de conocimientos, destrezas, capacidades, saberes, habilidades, conductas, aptitudes; es decir competencias relacionadas con la información". Se comenzò a usar el sintagma alfabetización informacional a partir de la propuesta de Paul Zurkowski para la National Commission on Libraries and Information Science (NCLIS), en 1974 (MIRANDA, et al, 2005).

Las competencias informacionales,

[...] consisten en poner en acción, en práctica, en juego, en movilización; de forma combinada, mezclada o integrada; en un contexto y con un contenido determinado (transferibles), todos los recursos (habilidades, conocimientos, actitudes); para solucionar con éxito problemas y aprender a aprender, a partir de la interacción efectiva con la información; sin delimitación de tipo, formato y soporte. Son necesarias en cualquier ámbito e incluyen otras competencias como las tecnológicas, las bibliotecarias, las bibliográficas, el pensamiento crítico y las sociales (DIAS SANCHEZ, 2008, p. 113).

Los objetivos de un plan de acción para mejorar el papel de las bibliotecas públicas, los museos y archivos fue establecido en 2003 en el Manifesto de Oeiras (DÍAZ-SÁNCHEZ, 2008). A continuación con la Declaración de Praga en el mismo año como resultado de la Reunión de Expertos sobre Alfabetización Informacional, que fue organizada por la U.S. National Comission on Library and Information Science y el National Forum on Information Literacy, cuyo el lema fue "Hacia una sociedad alfabetizada en información" (DECLARACIÓN..., 
2003). Más tarde en 2005 con la Declaración de Alejandría, se proclamo que "la alfabetización informacional y el aprendizaje a lo largo de la vida son los faros de la sociedad de la información” (DECLARACIÓN..., 2005). Y en 2006 surge la Declaración de Toledo resultado del Seminario de Trabajo celebrado en Toledo (España) "Biblioteca, aprendizaje y ciudadanía: la alfabetización informacional (DECLARACIÓN..., 2006).

El movimiento de alfabetización informacional se consolidó en España en el ámbito bibliotecario con la constitución, en 2007, de un Grupo de Trabajo sobre Alfabetización Informacional que incluía representantes de diversos sectores públicos como el Ministerio de Cultura, el Ministerio de Educación y Ciencia, la Biblioteca Nacional, los departamentos con competencias en materia de bibliotecas las Comunidades Autónomas y la Red de Bibliotecas Universitarias pero no incluía representantes de la Universidad (HACÍA..., 2009).

Por supuesto, hay una estrecha relación entre la alfabetización informacional y el aprendizaje permanente, se entiende que una persona alfabetizada en información es la que tiene las competencias en información, aquella que ha aprendido a aprender. Las discusiones acerca del tema no se han agotado aún, pues los muchos documentos, manifiestos, declaraciones, foros y eventos de un modo general ofrecen distintos puntos de interés.

La Unesco en su Manifiesto de 1949 ya consideraba el aprendizaje como uno de sus objetivos. Con el paso del tiempo, la llegada continua de inovaciones tecnológicas y las nuevas posibilidades de acceso a la información y aún el reconocimiento al derecho a la información, los servicios en las bibliotecas públicas han cambiado para satisfacer estas nuevas demandas. La IFLA/Unesco publica en 1994 un nuevo Manifiesto donde propone que las bibliotecas públicas tengan doce misiones y en dos de llas hay una preocupación respecto al aprendizaje: "Misión 2: apoyar tanto la educación individual y autodirigida como la educación formal a todos los niveles. Misión 11: facilitar el desarrollo de habilidades informacionales y digitales" (MANIFESTO..., 1994).

Batt (1998) apunta que las bibliotecas públicas pueden ayudar a sus usuarios en las más variadas formas de aprender, como en los aspectos relativos a aprender a aprender y a vivir y en los aspectos relativos al aprendizaje permanente. El autor denuncia que hay una creencia generalizada en que el aprendizaje se hace a través de las estructuras formales de aprendizaje. El aprendizaje informacional es una premisa de la sociedad de la información y del conocimiento, como indica Suaiden (2012, p. 73) "en la sociedad de la información la riqueza se ve favorecida por la producción de conocimiento". Y por su parte la biblioteca pública es el lugar de producción de conocimiento por excelencia.

\section{Acceso a la información y el compromiso social de las bibliotecas públicas}

Las herramientas de acceso a la información son fundamentales para el fortalecimiento de las competencias informacionales en las bibliotecas públicas y especialmente para combatir la brecha digital. En este sentido y siguiendo a Cuevas-Cerveró y Simeão (2011, p. 56) la "alfabetización informacional es una parte esencial de la inclusión digital" y por supuesto la responsabilidad de las bibliotecas públicas para el efectivo acceso a la información en la era digital es muy grande. Ante las demandas de la sociedad de la información y del conocimiento la biblioteca pública desempeña un papel muy relevante para la democratización y el acceso a la información. Planteando las responsabilidades de la biblioteca pública en la sociedad Froud y Mackenzie (2003, p. 26) afirman que "sin embargo, el principal desafío de las bibliotecas seguirá siendo el acceso a la información". Además añaden que "[...] las bibliotecas públicas han de desempeñar un papel decisivo en la creación de comunidades de aprendizaje" (FROUD; MACKENZIE, 2003, p. 32).

De acuerdo con Cuevas-Cerveró y Simeão (2011, p. 57) al apuntar que las "bibliotecas nacen para las necesidades sociales, son mantenidas por la sociedad, y permanecen para el uso de la sociedad", señalamos que al tratarse de las transformaciones sociales que ha sufrido la biblioteca pública en la sociedad actual y el desarrollo económico, político y social que las acompaña, estas instituciones no se han mantenido ajenas a esta cuestión y han experimentado profundas transformaciones para convertirse en el centro de información por excelencia para la comunidad en la que se encuentra

Al tratar de bibliotecas públicas debemos reconocer su contribución en dos cuestiones esenciales: entender la información como un derecho y los aspectos relacionados con el uso de internet y el acceso a la información. Sobre este punto Cuevas-Cerveró (2009, p. 201) expone que "[...] las bibliotecas públicas sobresalen por su contribución a materializar los derechos que la Constitución reconoce a los ciudadanos: el derecho a la educación, el derecho de acceso a la cultura y el derecho a la información". Por su concepto de institución social, abierta y democrática las bibliotecas públicas se convierten en instituciones para el aprendizaje continuo 
y para el desarrollo de competencias informacionales. Esto significa el reconocimiento de nuevos conceptos y funciones que al mismo tiempo suponen nuevas necesidades de información y nuevas formas de trabajar.

Atendiendo a lo expuesto la Internet en las bibliotecas públicas cobra una gran importancia pues ayuda a garantizar a los usuarios de la biblioteca pública el acceso a la información sin estar determinados por espacios físicos. Giappiconi; et al (2001) afirman que se trata de una preocupación muy destacada en las bibliotecas públicas en diversos países desde el comienzo y mediados de los años noventa. De acuerdo con los autores, "Proporcionar a los ciudadanos un acceso general a internet, constituye uno de los principales servicios de todas las bibliotecas públicas" (GIAPPICONI; et al, 2001, p. 34).

Internet es una herramienta esencial en las bibliotecas públicas de nuestros días. El uso de la internet en esta institución de carácter público garantiza a los ciudadanos el acceso a uno de sus derechos sociales más básicos, el derecho a la información. Esto significa que el compromiso social de la biblioteca pública es en primera instancia con sus usuarios.

\section{Procedimientos metodológicos}

La investigación es un proceso de búsqueda de conocimiento basada en la ciencia teórica-conceptual y sigue un modelo sistemático. En este sentido, esta investigación, de carácter exploratorio, analiza la aplicación de metodologías de alfabetización en información e inclusión digital y social en la sociedad de la información en Brasil y España, observando particularmente los aspectos relacionados con la interactividad y la conectividad en las bibliotecas públicas de los dos países como supuestos necesarios para la sociedad de la información.

El análisis de los datos incluye aspectos cualitativos y cuantitativos a través de las informaciones contenidas en los sitios institucionales de la red de bibliotecas públicas de los dos países y en la literatura sobre el tema. El método de enfoque y razonamiento es dialéctico y permite comprobar con mayor rigor los objetivos del análisis, por tratarse de un método dinámico y interpretativo (GIL, 1999).

\section{Resultados}

Los resultados preliminares del análisis de los datos fueran recolectados en septiembre y octubre a través de un formulario survey de investigación enviado por correo eletrónico y consulta en los sites de cada institución de cada país. En Brasil se consultó la home page del Sistema Nacional de Bibliotecas Públicas (SNBP/RJ) ${ }^{1}$ y en España el site del Ayuntamiento y la Red de Bibliotecas Públicas de España ${ }^{2}$. El corpus está compuesto por 27 bibliotecas públicas pertenecientes a diferentes estados de Brasil y 154 bibliotecas públicas de siete comunidades autónomas en España: Madrid, Asturias, Islas Balears, Murcia, Navarra, Cantabria y La Rioja.

\subsection{Interactividad y conectividad en las bibliotecas públicas de Brasil}

El SNBP/RJ, en consonancia con el proyecto Sociedad de la Información (Soclnfo) puesto en marcha por el Gobierno en diciembre de 1999, creó un documento de acceso universal a la información y lucha contra la desigualdades, en el que propuso desarrollar un programa de acciones para situar a las bibliotecas públicas de acuerdo con los objetivos del proyecto Sociedad de la información ${ }^{3}$ Esto implicaría tanto la coordinación de SNPB/RJ como todo el país e incluye: capacitación de los recursos humanos y capacitación de los usuarios; consorcio entre bibliotecas electrónicas, automatización y conexión electrónica de las bibliotecas, acceso y la conectividad a internet y las redes de alta velocidad, creación de sítios de cada biblioteca, consistente en el desarrollo regional y la creación de um núcleo para las colecciones digitales; programa de registro, organización y gestión de la información e identificación de los proveedores de las agencias y de los recursos. El objetivo era establecer una red de bibliotecas digitales, conectados a Internet 2.0, red de alta velocidad, lo que permitiría alto rendimiento de los procesos, la videoconferencia y directorios distribuidos, garantizando la calidad, la estabilidad, la seguridad y solidez de los servicios prestados a través de Internet.

Presentamos un análisis de las bibliotecas públicas brasileñas y sus posibilidades de conectividad y interactividad mediada por internet. El gráfico que presentamos a continuación permite visualizar mejor los datos: 


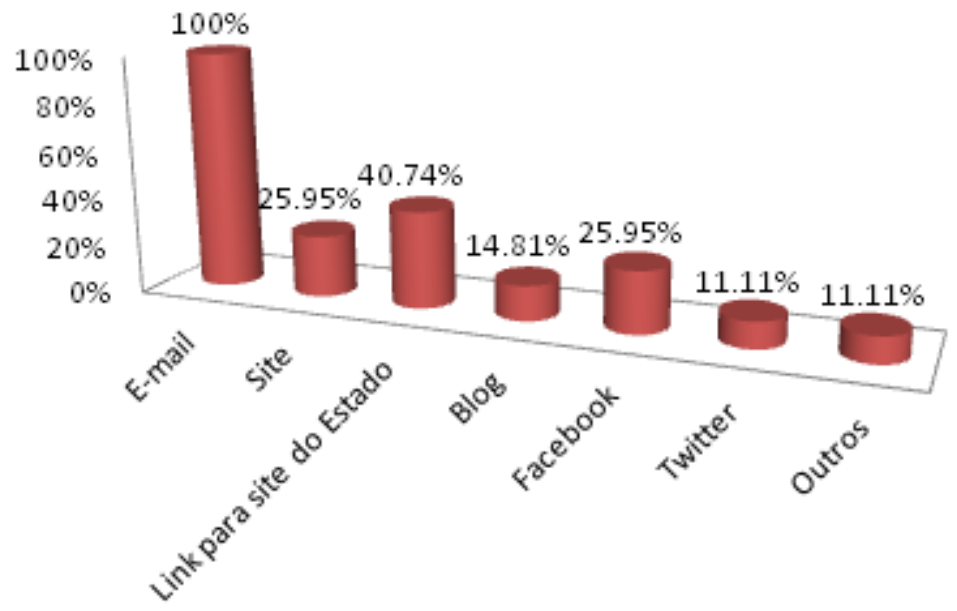

Gráfico 1 : Interactividad de las Bibliotecas Públicas Brasileñas

Más de diez años después de la creación del Proyecto Sociedad de la Información en Brasil, poco más del 50\% de las bibliotecas están en internet. La participación de las bibliotecas en las redes sociales es pequeña, sin embargo, todas las bibliotecas tienen correo electrónico para hacer preguntas y comunicarse con la gestión de las bibliotecas. La conectividad en las bibliotecas públicas se investigó a través de formulario survey con once respuestas como se muestra en el grafico abajo:

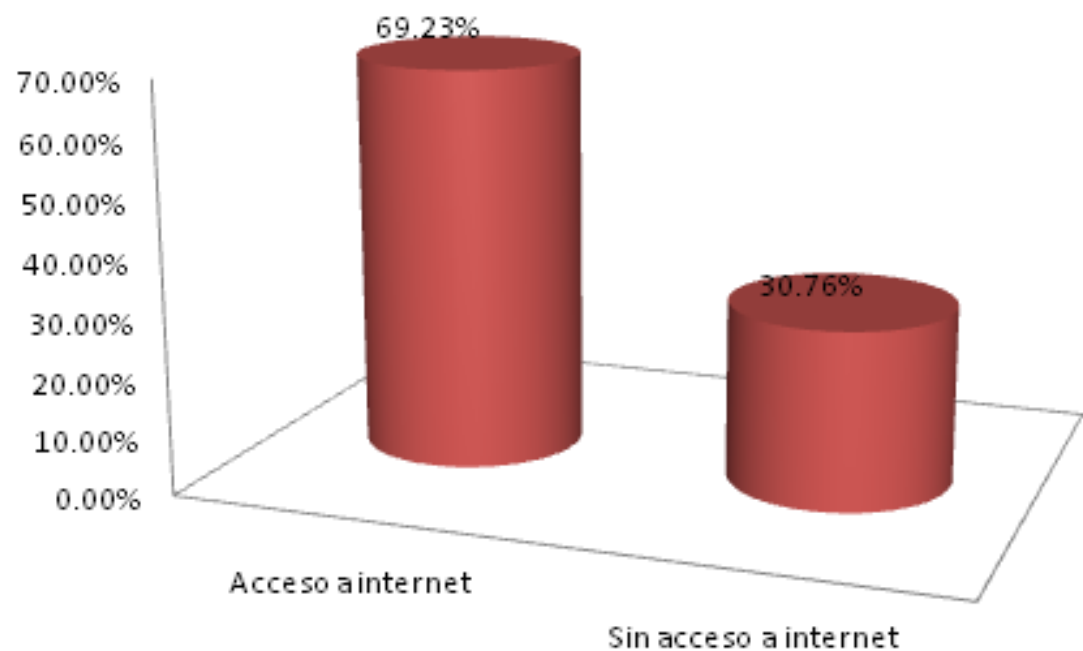

Gráfico 2 : Conectividad de las Bibliotecas Públicas Brasileñas

Se consideró, para efectos de conectividad, a las bibliotecas públicas que tenían al menos un ordenador con acceso a Internet para los usuarios disponible. Los datos muestran que a pesar de caminar a paso lento, Brasil camina hacia una democratización del acceso a la información en sus bibliotecas públicas. Sin embargo, debemos destacar que las bibliotecas que se investigaron son las bibliotecas públicas del Estado que como coordinadoras de los sistemas estatales de bibliotecas públicas deben estar de acuerdo con los presupuestos de la sociedad de la información.

\subsection{Interactividad y conectividad en las bibliotecas públicas de España}

Las bibliotecas públicas de España presentan respecto a internet y la realidad en este ámbito una trayectoria ya muy consolidada reflejada en su visibilidad en la red para el mundo. El acceso a Internet en las bibliotecas públicas de España es fruto de un proyecto del gobierno y de una planificación por parte de la administración bibliotecaria. Su implantación ha dependido de la voluntad de cada gobierno municipal y comunidades autónomas. 
En 2000 la implantación del Plan de Impulso de las Bibliotecas Públicas Españolas propuso un nuevo modelo de biblioteca pública como centro de acceso al conocimiento, independientemente de su soporte material y de su ubicación física, y de utilidad para todos los ciudadanos. De acuerdo a las necesidades en la sociedad de la información el plan propone las siguientes funciones: a) ofrecer acceso a la información electrónica disponible en Internet y soportes digitales; b) dar a los usuarios la formación necesaria en el uso de las tecnologías de la información y comunicación, ayuda y orientación; e c) Contribuir a preservar y contribuir el patrimonio cultural y local, convirtiéndose en proveedoras de información local a través de Internet ${ }^{4}$.

La presencia de las bibliotecas públicas de España em la web es muy fuerte, prácticamente el $100 \%$ de las bibliotecas públicas tienen sitio web y están ofreciendo servícios de préstamo de libros electrónicos y dispositivos de lectura electrónico, conforme apunta Goméz-Goméz (2012, p. 6): "Por el momento, la realidad es que son muchas las bibliotecas públicas españolas que ya vienen ofreciendo dispositivos de lectura (e-readers) de libros electrónicos (e-books) en préstamo a sus usuários". Como se observa en el gráfico abajo casi la totalidad de las bibliotecas públicas en España están en la internet.

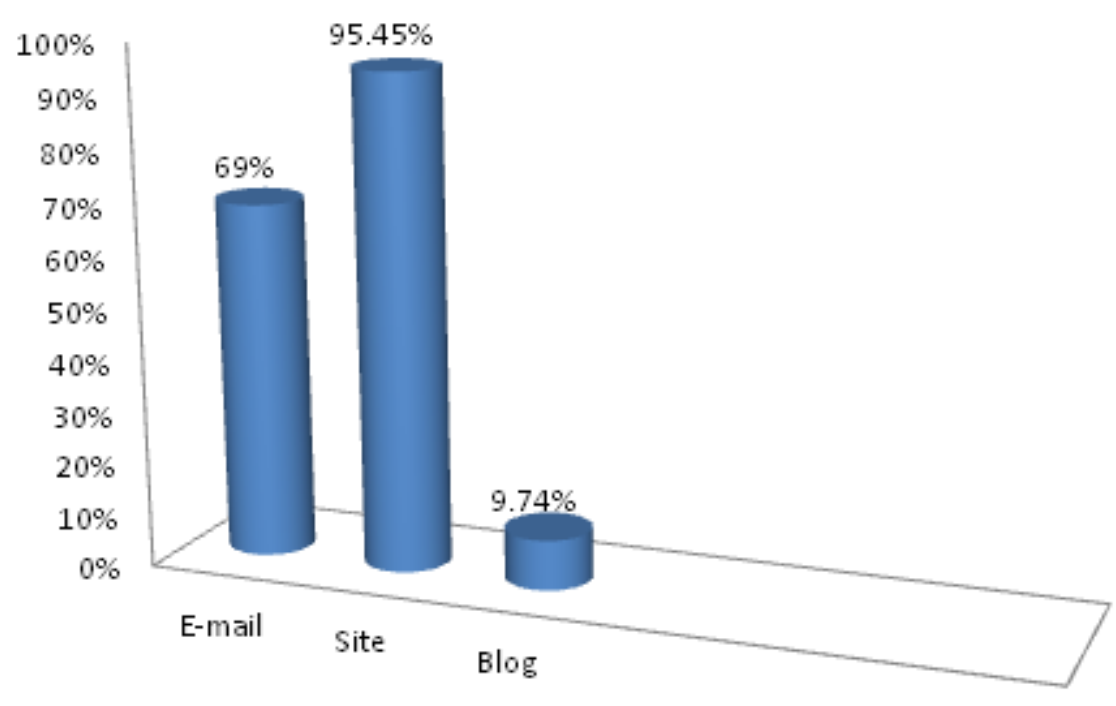

Gráfico 3 : Interactividad de las Bibliotecas Públicas Españolas

Al visitar el site no se observó ningún indicio de las redes sociales, por tratarse de una home page institucional y padronizada para todas las bibliotecas. Encuentramos sólo la presencia de los blogs. Todavia en relación a la conectividad, 95\% de las bibliotecas públicas tienen acceso a internet a los usuarios, como muestra el grafico 4:

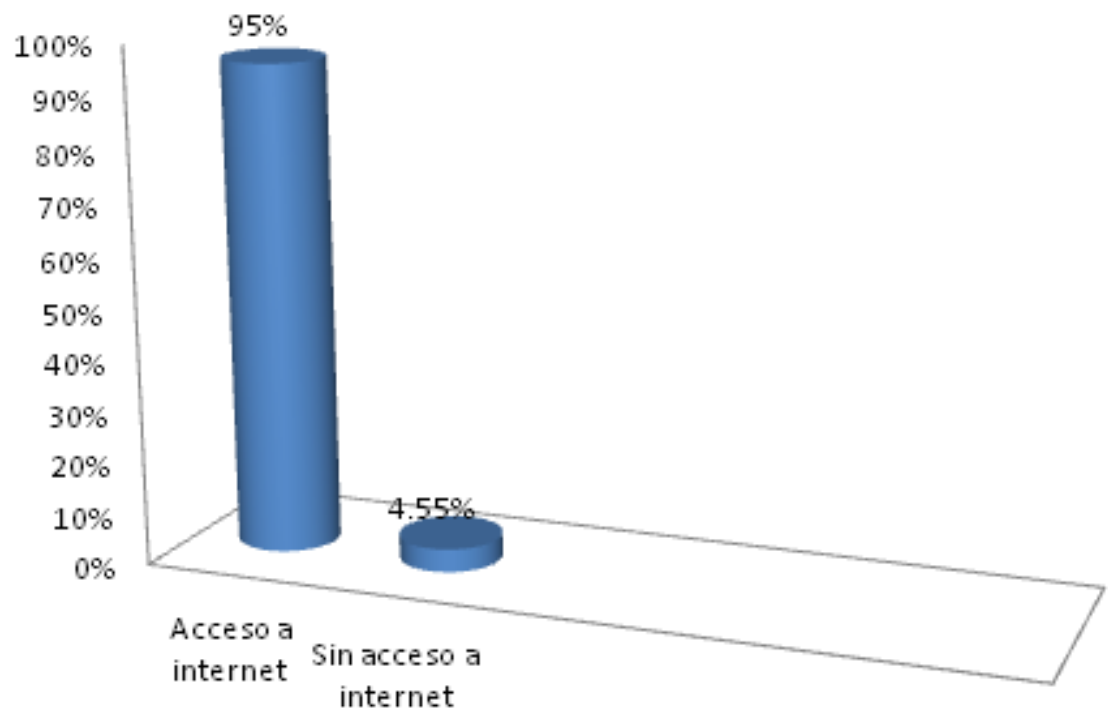

Gráfico 4 : Conectividad de las Bibliotecas Públicas Españolas 
Sin duda, asegurar el desarrollo de la Sociedad de la Información consiste en asegurar la democratización del acceso a la información. López-Marin (2002, p. 4) afirma que "las bibliotecas públicas han representado, desde siempre, esa democratización del acceso a la información que ahora descansa de forma masiva en las Tecnologías de la Información y de la Comunicación (TIC)". Además las políticas de información actuales fomentan el acceso gratuito a la información por todos los ciudadanos.

\subsection{Comparación de interactividad y conectividad de las bibliotecas públicas de Brasil y España}

El gobierno brasileño creó en 2002 y revisó en 2008 el Programa de Gobierno Electrónico - Servicio al Ciudadano (GESAC), que comenzó a dar prioridad a la conectividad en las bibliotecas públicas, telecentros comunitarios, escuelas, comunidades quilombolas, pueblos indígenas y areas fronterizas/selva $\mathbf{5}$.

Ya en España el gobierno creó en 2000 el Plan de Impulso de las Bibliotecas Públicas, cuya finalidad era contribuir al desarrollo de las bibliotecas públicas españolas, como servicios públicos dirigidos a todos los ciudadanos que procuran garantizar el ejercicio del derecho a la información, la cultura y la lectura, y afianzar su posicionamiento en la sociedad de la información ${ }^{6}$.

El resultado de estas políticas, como se muestra en el siguiente gráfico es una diferencia de $26,22 \%$ entre los dos países.

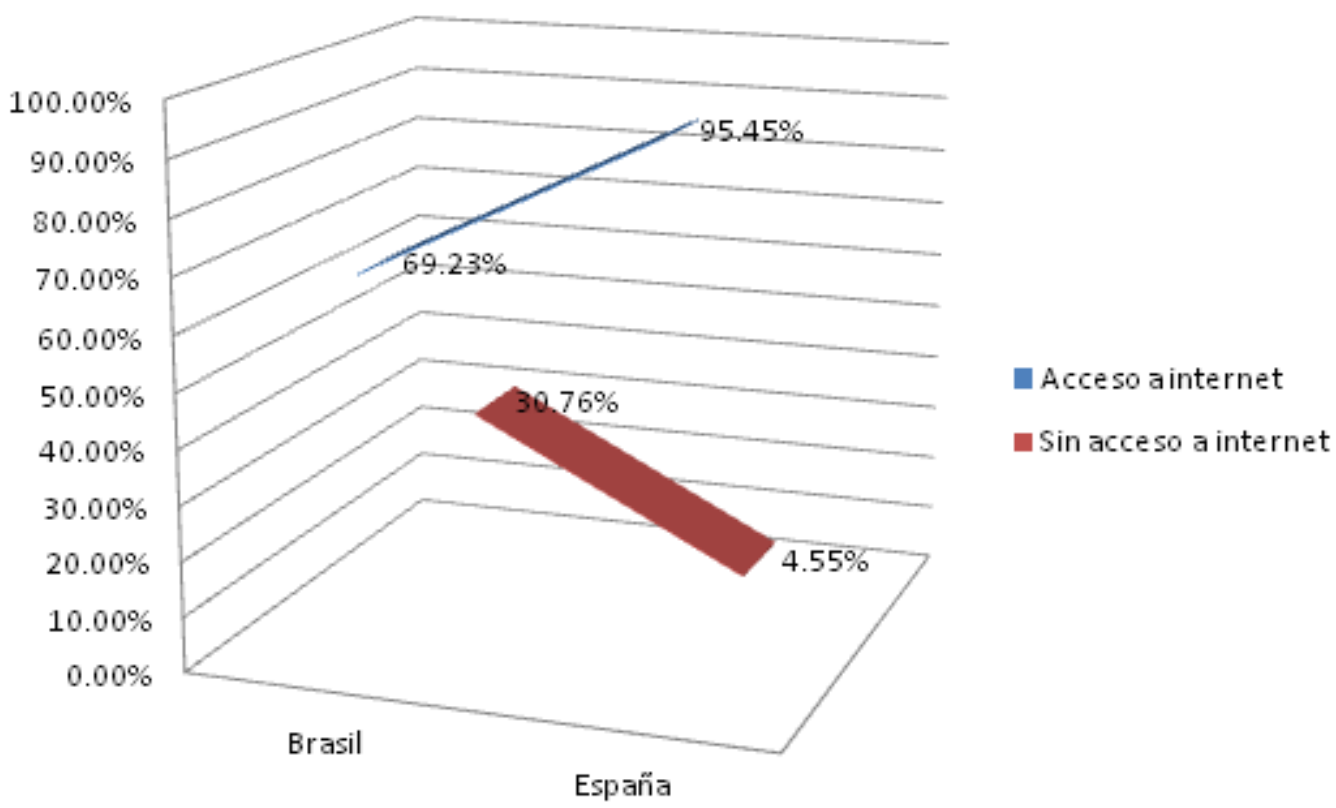

Gráfico 5 : Comparación de la conectividad entre Brasil y España

La comparación entre los dos países encuentra aún una gran brecha y apunta a una focalización más eficaz de las políticas públicas en Brasil con el fin de resolver estos problemas. Felicié Soto (2006) dice que las bibliotecas públicas deben servir de puente entre las personas y la sociedad de la información. Para tal apunta que "la automatización de las bibliotecas, la conexión a internet y el acceso a redes de información telemáticas son elementos cruciales para enfrentar los efectos de la brecha digital” (FELICIÉ SOTO, 2006, p. 117).

\section{Conclusiones}

Es importante entender que el acceso a las Tecnologías de Información y Comunicación, la producción de contenidos y la mejora de la infraestructura de información - representada en la modernización y conectividad de las bibliotecas públicas -, depende em gran medida de la adopción de políticas públicas pertinentes. 
En Brasil, según el Instituto Brasileño de Geografía y Estadística (IBGE) ${ }^{7}$, sólo $12,46 \%$ de la población tiene acceso a los ordenadores, de los cuales sólo El 8,31\% están conectados a Internet. Todavía la biblioteca pública no ha sido en absoluto ajena a esta nueva realidad e intenta adaptarse a la revolución digital, a la interactividad y conectividad que sólo es el principio de la Era de la Información y del Conocimiento. Los datos del primer Censo Nacional de Bibliotecas Públicas Municipales hecho por la Fundación Getúlio Vargas (FGV) para la Fundación Biblioteca Nacional (FBN) en 2010, apunta que hay 4.763 bibliotecas públicas en el Brasil, de las cuales $45 \%$ tienen ordenadores y sólo $17 \%$ disponen de wifi para los usuarios ${ }^{8}$. Las estadísticas españolas son más positivas hacia la conectividad. Hay en España 4.164 bibliotecas públicas, de las cuales, 89,57\% tienen ordenadores y $93,82 \%$ de las bibliotecas tienen acceso a internet ${ }^{9}$.

Moore (1998) reconoce además que el papel de las bibliotecas públicas será cada vez más importante en la sociedad del conocimiento, asumiendo que recaen en ellas muchas de las competencias del derecho fundamental a la información. En este sentido, comprender la concepción de la biblioteca pública como una institución democrática que se relaciona directamente con el derecho a la información y el acceso libre al conocimiento es una prioridad. Así el papel de las bibliotecas públicas en Internet es crucial para garantizar el acceso universal a la información.

\section{Referencias}

AZEVEDO, M; GOUVEIA, L. B. (2003). Repensar a missão da biblioteca pública na gestão das TIC. In: CONFERÊNCIA IBERO-AMERICANA, 1. Algarve, Portugal, 8 al 9 de noviembre, Anales eletrónicos... Algarve, Portugal: ladis. Disponíble en: http://www.iadis.net/dl/final uploads/200303L015.pdf Acceso en: 13 oct. 2012.

BATT, C. (1998). I have seen the future and It works. Ariadne: web magazine for information professionals, n. 18. Disponible en: http://www.ariadne.ac.uk/issue18/batt Acceso en: 13 oct. 2012.

COUTO, L. M. M. (1988). A produção da informação e o MEC. Revista de Biblioteconomia de Brasilia, Brasília, v. 16, n. 1, p. 65-74, jan.,/jun.. Disponíble en: www.brapci.ufpr.br/download.php?dd0=17648 Acceso en: 12 oct. 2012.

CUEVAS-CERVERÓ, A; SEMEÃO, E. (Coord.). (2011). Alfabetización informacional e inclusión digital: hacia un modelo de infoinclusión social. Gijón (España): Ediciones Trea.

CUEVAS-CERVERÓ, A. (2009). Formación de la ciudadanía en entornos de información electrónica: alfabetización informacional. In: VIVES, J. (Coord.) Digitalización del património: archivos, bibliotecas y museos en la red. Barcelona: Editorial UOC.

DECLARACIÓN de Praga: Hacia una sociedad alfabetizada en información. (2003). Disponible en: www. alfinred.org/blog/2005/contenido/40 Acceso en: 13 oct. 2012.

DECLARACIÓN de Alejandría sobre la alfabetización informacional y el aprendizaje a lo largo de la vida: "Faros para la Sociedad de la Información". (2005). Formulada en el Coloquio de Expertos de Alejandría, de 6-9 noviembre, organizado por Unesco y el National Forum on Information Literacy. Disponible en: http://www. ifla.org//ll/wsis/BeaconlnfSoc-es.html Acceso en: 13 oct. 2012.

DECLARACIÓN de Toledo sobre alfabetización informacional: Bibliotecas por el aprendizaje permanente. (2006). Y resto de las actas del Seminario. Disponible en: http://travesia.mcu.es/S ALFIN/index.html Acceso en: 13 oct. 2012.

DÍAZ SÁNCHEZ, M. (2008). Las competencias desde la perspectiva informacional: apuntesintroductorios a nivel terminológico y conceptual, escenarios e iniciativas. Ci.Inf., Brasília, v.37, n.1, p. 107-120. Disponíble en: http://revista.ibict.br/ciinf/index.php/ciinf/article/view/935 Acceso en: 13 oct. 2012.

FELICIÉ SOTO, A. M. (2006). Biblioteca pública, sociedade de la información y brecha digital. Buenos Aires: Alfagrama.

FROUD, R; MACKENZIE, C. (2003). Gobierno electrónico y bibliotecas públicas: impulse a la información local: Barcelona: Fundación Bertelsmann.

GIAPPICONI, T; et al. (2001). Servícios de future basados en internet en las bibliotecas públicas: una investigación con ejemplos. Barcelona: Fundación Bertelsmann.

GIL, A. C. (1999). Métodos e técnicas de pesquisa social. São Paulo: Atlas. 
GOMÉZ-GOMÉZ, A. A. (2012). La realidad y el deseo en la prestación de servicios digitales en bibliotecas: la experiencia de la biblioteca del Estado en Huelva. CONGRESO NACIONAL DE BIBLIOTECAS PÚBLICAS, 6. Burgos, 9 al 11 de octubre. Anales... Burgos: MCU.

HACÍA la alfabetización informacional en las bibliotecas públicas españolas: informe del grupo de trabajo de alfabetización informacional. (2009). Madrid: Consejo de Cooperación Bibliotecária. Disponíble en: http://www.mcu.es/bibliotecas/MC/ConsejoCB/gt alfin/ALFIN en BP 2009.pdf Acceso en: 13 oct. 2012.

JARAMILLO, O; et al (2008). La biblioteca pública y su gestión: en el contexto de la sociedade de la información. Buenos Aires: Alfagrama.

LÓPEZ-MARIN, L; et al. (2002). Evaluación de la accesibilidad y usabilidad de los sitios web de las bibliotecas públicas catalanas. Revista de biblioteconomia i documentació, n. 31, p. 17-51, maio/ago. Disponíble en: http://sid.usal.es/idocs/F8/ART6497/accesibilidad usabilidad.pdf Acceso en: 29 oct. 2012.

MANIFESTO DA IFLA/UNESCO sobre bibliotecas públicas. (1994). Disponíble en: http://archive.ifla.org/VII/s8/unesco/port.htm Acceso en: 7 sep. 2012

MARTíNEZ, J. (2002). Retos de la biblioteca pública en la sociedad de la información. CONGRESO NACIONAL DE BIBLIOTECAS PÚBLICAS, 1. Valencia, 29 a 31 de octubre. Anales eletrónicos... Valencia, MCU, 2002. Disponíble en: http://www.mcu.es/bibliotecas/docs/Cooperacion/CongresoNacionalBP 01.pdf Acesso em 12 oct. 2012.

MIRANDA, A; et al. (2005). Telecentros do governo: inclusão e alfabetização digital. In: Encontro de Arquivos e Bibliotecas da Administração Pública, 3; Seminário Nacional de Comutação Bibliográfica, 3. Brasília, 20 a 23 de septembre. Anales... Brasília: IBICT. Disponible en: http://www.antoniomiranda.com.br/CInformacao.htm Acceso en: 13 oct. 2012.

(1978). A missão da biblioteca pública no Brasil. Brasília: MEC/Capes.

MOORE, N. (1998). Rights and Responsibilities in an Information Society. Journal of Information Law and Technology (JILT), $\mathrm{n}$ 1. Disponible en: http://www2.warwick.ac.uk/fac/soc/law/eli/iilt/1998 1/moore/ Acceso en: 30 oct. 2012.

SEOANE-GARCIA, C. (2012). Reconsquistar a usuários, enamorar a ciudadanos y dialogar com personas: las bibliotecas municipais da Coruña y las redes sociales. CONGRESO NACIONAL DE BIBLIOTECAS PÚBLICAS, 6. Burgos, 9 al 11 de octubre. Anales... Burgos: MCU.

SUAIDEN, E. J. (2012). A alfabetização informativa e a inclusão na sociedade da informação. In: HERNÁNDEZ SALAZAR, P. Tendencias de la alfabetización informativa en Iberoamérica. México: UNAM/Centro Universitario de Investigaciones Bibliotecológicas. Disponíble en: http://132.248.242.3/ publica/archivos/libros/tendencias alfabetizacion informativa.pdf Acceso em: 13 oct. 2012. 


\title{
Datos de los autores
}

Maria Cleide Rodrigues Bernardino

Professora na Universidade Federal do Cariri (UFCA). Centro de Ciências Sociais Aplciadas (CCSA). Doutora em Ciência da Informação pela Universidade de Brasília.

cleide.rodrigues@ufca.edu.br

\section{Aurora Cuevas-Cerveró}

Professora na Universidad Complutense de Madrid. Doutora em Ciências da Documentação pela Universidad Carlos III de Madrid.

auro.cuevas@gmail.com

\section{Emir José Suaiden}

Professor na Universidade de Brasília. Departamento de Ciência da Informação. Doutor em Ciência da Informação pela Universidad Complutense de Madrid. Pós-Doutor pela Universidad Carlos III de Madrid.

emir@unb.br

\author{
Recibido - Received : 2014-02-26 \\ Aceptado - Accepted : 2014-12-30
}

\footnotetext{
${ }^{1}$ VER: www.bn.br/snbp/

${ }^{2}$ VER: http://www.bibliotecaspublicas.es

${ }^{3}$ VER: www.bn.br/snbp/Textos/socinfo.doc

${ }^{4}$ VER: http://www.diba.es/biblioteques/documentspdf/MinisteriPla2000.PDF

5 VER: https://i3gov.planejamento.gov.br/textos/livro4/4.6_Inclusao_Digital.pdf

${ }^{6}$ VER: http://www.diba.es/biblioteques/documentspdf/MinisteriPla2000.PDF

${ }^{7}$ VER: http://www.ibge.gov.br/home/presidencia/noticias/noticia_visualiza.php?id_noticia=1455\&id_pagina

${ }^{8}$ VER: http://www.cultura.gov.br/site/wp-content/uploads/2010/05/microsoft-powerpoint-fgv-ap-minc-completa79.pdf

${ }^{9}$ VER: http://www.mcu.es/culturabase/cgi/axi
}

\section{(c) EY EY}

This work is licensed under a Creative Commons Attribution 4.0 United States License.

\section{UILIS D-Sonfe?}

This journal is published by the University Library System of the University of Pittsburgh as part of its D-Scribe Digital Publishing Program and is cosponsored by the University of Pittsburgh Press. 